\title{
Surveillance system of vaccine adverse events and local data analysis - the experience in a middle-sized city in Brazil, 1999-2001
}

\author{
Guilherme Côrtes Fernandes ${ }^{\mathrm{a}, \mathrm{b}, *}$, Luiz Antonio Bastos Camacho ${ }^{\mathrm{b}}$, Marilia Sá Carvalho ${ }^{\mathrm{b}}$ \\ a Santa Casa de Misericórdia de Juiz de Fora, Minas Gerais, Brazil \\ ${ }^{\mathrm{b}}$ Fundação Oswaldo Cruz, Escola Nacional de Saúde Pública, Departamento de Epidemiologia e Métodos Quantitativos em Saúde, \\ Rua Leopoldo Bulhões, 1480 sala 820, Manguinhos, Rio de Janeiro, Cep 21041-210, RJ, Brazil
}

Available online 18 January 2005

\begin{abstract}
We reviewed all vaccine adverse events (VAE) notified in a middle-sized Brazilian city $(n=247)$ to the National Immunisation Program between January 1999 and December 2001. Vaccine doses used in that period were considered for rate estimates. Aspects of the surveillance system (SS) and their influences on collected data were considered, searching for contributions of local data analysis to investigation of VAE and to the monitoring of vaccine safety. Notification rates in our study were higher when compared to national data. Changes in the notification pattern were observed following vaccination campaign periods. An increase in aseptic meningitis cases temporally associated to yellow fever vaccine was detected. The analysis of local data provided information unperceived in national consolidated data. Through this analysis we detected: events related to application technique and handling; people's perception changes on VAE; and the local SS's ability to raise new hypothesis. We suggested changes to the notification form regarding data entry criteria and analysis.
\end{abstract}

(C) 2005 Elsevier Ltd. All rights reserved.

Keywords: Vaccine; Adverse events; Surveillance

\section{Introduction}

Vaccine adverse events (VAE) are a growing concern in decision-making processes regarding vaccine use in individuals and populations. The fast identification and investigation of VAE is important so that a clear and scientifically supported response can be offered to the population $[1,2]$. Available data on reactogenicity of vaccines currently used in public health are sparse due to the low incidence of cases and to limitation of the VAE surveillance systems [1,2], making it difficult to identify events and raise hypothesis [3]. In spite of its limitations, this passive surveillance system is important as an ongoing awareness system for events genuinely caused by vaccines and for false alarms that could

\footnotetext{
* Corresponding author. Tel.: +55 3232347387/2125982630; fax: +55 3232347387.

E-mail addresses: gcortes@ riscobiologico.org (G.C. Fernandes), luiz.camacho@ensp.fiocruz.br (L.A.B. Camacho).
}

interfere in the general public acceptance of vaccination. Additional clinical and epidemiological investigations are frequently necessary to clarify hypothesis raised by surveillance data [4-7].

In Brazil, the VAE passive surveillance system is coordinated by the National Immunisation Program (PNI), data are generated at local levels by vaccination units and recorded in standard forms for notification of suspected cases [8].

The analysis of local data collected in a middle-sized city-approximately 500,000 inhabitants-with well structured vaccine and surveillance programs may provide information relevant to the functioning of the surveillance system impossible to detect in consolidated data at national level. The aim of this study is to contribute to the understanding of the kind of information that can be obtained by a VAE passive surveillance system, highlighting the limitations and possibilities of local data analysis. The local system's functionality and ability to detect VAE, as well as the possibility of answering questions and raising hypothesis are discussed. 


\section{Materials and method}

A cross-sectional study was performed based on VAE notified cases recorded by public and private vaccination units in the city of Juiz de Fora, Brazil, from January 1999 to December 2001. The totals of doses administered in vaccination campaigns, as well as the campaigns dates, were collected in a record keeping book and served as denominators for calculating notification rates. We analysed all the variables available in the PNI notification forms, considering data quality, as well as the characteristics of the forms used, methodologic issues and service practice. The frequency and distribution of locally notified VAE was compared with data from the whole country released by PNI.

\section{Results}

Of 247 notification forms for the period, 19 were excluded from analysis due to double notification (12 cases), VAE from 1998 ( 2 cases), non-VAE (5 cases). Half of the notifications analysed were made in 2001 and $72.0 \%$ of total notification involved diphteria toxoid - tetanus toxoid - pertussis (DTP), yellow fever (YF), BCG, and diphteria toxoid - tetanus toxoid (dT) vaccines (Table 1). In 2001, YF vaccine was involved in $41.0 \%$ of VAE, largely outnumbering DTP and BCG vaccines. $56.1 \%$ of all notifications involved female subjects, $39.0 \%$ were male subjects, and in $4.8 \%$ gender was not informed. The highest proportion of VAE occurred in children under one year old (31.1\%) and in the 15-59 year-old group $(30.5 \%$ ) (Table 2). $56.4 \%$ of VAE in children under 5 years old were attributed to DTP vaccine, whereas in individuals aged 5 years or more, $47.6 \%$ of VAE involved YF vaccine.
Table 2

Distribution of VAE per vaccine and age

\begin{tabular}{|c|c|c|c|c|c|c|}
\hline \multirow[t]{2}{*}{ Vaccine $^{\mathrm{a}}$} & & \multicolumn{5}{|c|}{ Year of age } \\
\hline & & $<1$ & $1-5$ & $5-15$ & $15-59$ & $>60$ \\
\hline \multirow[t]{2}{*}{ BCG } & $N$ & 17 & 1 & 3 & 3 & - \\
\hline & $\%$ & 26.6 & 2.7 & 14.3 & 4.8 & - \\
\hline \multirow[t]{2}{*}{ DPT } & $N$ & 35 & 22 & 1 & - & - \\
\hline & $\%$ & 54.7 & 59.5 & 4.8 & - & - \\
\hline \multirow[t]{2}{*}{ Tetanus-diphteria } & $N$ & - & - & - & 10 & 7 \\
\hline & $\%$ & - & - & - & 15.9 & 33.3 \\
\hline \multirow[t]{2}{*}{ Yellow fever } & $N$ & 1 & 3 & 9 & 31 & 3 \\
\hline & $\%$ & 1.6 & 8.1 & 42.9 & 49.2 & 14.3 \\
\hline \multirow[t]{2}{*}{ Measles } & $N$ & 2 & - & - & - & - \\
\hline & $\%$ & 3.1 & - & - & - & - \\
\hline \multirow[t]{2}{*}{ MMR } & $N$ & - & 5 & - & 1 & - \\
\hline & $\%$ & - & 13.5 & - & 1.6 & - \\
\hline \multirow[t]{2}{*}{ Measles-rubella } & $N$ & - & 1 & 3 & 8 & - \\
\hline & $\%$ & - & 2.7 & 14.3 & 12.7 & - \\
\hline \multirow[t]{2}{*}{ Influenza } & $N$ & - & - & - & 1 & 6 \\
\hline & $\%$ & - & - & - & 1.6 & 28.6 \\
\hline \multirow[t]{2}{*}{ Other vaccines } & $N$ & 9 & 5 & - & - & - \\
\hline & $\%$ & 14.0 & 13.5 & - & - & - \\
\hline Total & & 64 & 37 & 21 & 63 & 21 \\
\hline
\end{tabular}

Juiz de Fora-MG, 1999-2001.

a The vaccine related to the VAE was informed in 206 cases.

All over the period studied, BCG vaccine presented the highest notification rate per applied doses (Table 3), although in a decreasing trend. The rate of DTP-related VAE was higher than all others in 2001. The rates significantly increased in 2001 for YF vaccine following a regional mass vaccination campaign, and for the measles-rubella vaccine, which was object of a nation-wide vaccination campaign that year. Global notification rates obtained in local data analysis

Table 1

Frequency of VAE per vaccine and year of notification

\begin{tabular}{|c|c|c|c|c|c|c|c|c|}
\hline \multirow[t]{3}{*}{ Vaccine } & \multicolumn{6}{|c|}{ Year of notification } & \multicolumn{2}{|c|}{ Total } \\
\hline & \multicolumn{2}{|c|}{1999} & \multicolumn{2}{|c|}{2000} & \multicolumn{2}{|c|}{2001} & \multirow[b]{2}{*}{$N$} & \multirow[b]{2}{*}{$\%$} \\
\hline & $N$ & $\%$ & $N$ & $\%$ & $N$ & $\%$ & & \\
\hline DPT & 22 & 34.9 & 11 & 20.4 & 27 & 24.3 & 60 & 26.3 \\
\hline Yellow fever & 4 & 6.3 & 2 & 3.7 & 46 & 41.4 & 52 & 22.8 \\
\hline BCG & 13 & 206 & 13 & 24.1 & 8 & 7.2 & 34 & 14.9 \\
\hline Tetanus-diphteria & 6 & 9.5 & 7 & 13 & 4 & 3.6 & 17 & 7.5 \\
\hline Measles-rubella & - & & 2 & 3.7 & 10 & 9 & 12 & 5.3 \\
\hline Influenza & 1 & 1.6 & 1 & 1.9 & 6 & 5.4 & 7 & 3.1 \\
\hline MMR & 3 & 4.8 & 2 & 3.7 & 2 & 1.8 & 7 & 3.1 \\
\hline Pneumococcus & - & & 4 & 7.4 & - & & 4 & 1.8 \\
\hline Hepatitis B & - & & 1 & 1.9 & 2 & 1.8 & 3 & 1.3 \\
\hline Oral polio (Sabin) & 1 & 1.6 & 1 & 1.9 & 1 & 0.9 & 3 & 1.3 \\
\hline Rabies & 1 & 1.6 & 1 & 1.9 & 1 & 0.9 & 3 & 1.3 \\
\hline Measles & 2 & 3.2 & - & & 1 & 0.9 & 3 & 1.3 \\
\hline Haemophilus & 1 & 1.6 & - & & - & & 1 & 0.4 \\
\hline Missed & 9 & 14.3 & 9 & 16.7 & 4 & 3.6 & 22 & 9.6 \\
\hline Total & 63 & 100.0 & 54 & 100.0 & 111 & 100.0 & 228 & 100.0 \\
\hline
\end{tabular}

Juiz de Fora-MG, 1999-2001. 
Table 3

VAE notification rates per 100,000 doses in Juiz de Fora (JF) (1999-2001) and in Brazil (PNI) ${ }^{\mathrm{a}}(2000-2002)$

\begin{tabular}{lccclc}
\hline & $1999 \mathrm{JF}$ & $2000 \mathrm{JF}$ & $2001 \mathrm{JF}$ & $1999-2001 \mathrm{JF}$ & PNI $^{\mathrm{a}}$ \\
\hline BCG & 110.7 & 91.7 & 53.5 & 83.3 & 4.4 \\
DPT & 60.3 & 31 & 71.6 & 54.7 & 10.7 \\
MMR & 34.3 & 18.8 & 22.4 & 24.7 & $14.1^{\mathrm{b}}$ \\
Rabies & 21.2 & 21.5 & 22.9 & 21.9 & 4.7 \\
Measles-rubella & - & 7.2 & 16.2 & 13.5 & $\mathrm{c}$ \\
Measles & 31.5 & - & 14.8 & 13.4 & $\mathrm{c}$ \\
Yellow fever & 9.2 & 3.1 & 13.4 & 11.5 & 0.6 \\
Tetanus-diphteria & 6.5 & 10.1 & 7.8 & 7.5 & 0.6 \\
Influenza & 3.4 & 2.5 & 15.5 & 6.2 & $\mathrm{c}$ \\
Hepatitis B & - & 2.7 & 1.3 & 1.3 & 0.2 \\
Oral polio & 0.9 & 0.9 & 0.9 & 0.9 & $\mathrm{c}$ \\
\hline
\end{tabular}

${ }^{a}$ National Immunization Program.

b Estimated rate, MMR vaccination campaign Rio de Janeiro, 1996.

${ }^{c}$ Data not available.

were higher than those found from PNI data. BCG-related VAE showed the higher discrepancy in notification rates: almost 20 times higher in Juiz de Fora where it ranked first, whereas for the whole country it ranked fourth. Proportions of VAE per vaccine in each age group revealed some important differences. In children under one year of age (data not shown) the proportion of BCG-related VAE was four times higher in local data analysis when compared to national data (32.8\% and $8.4 \%$, respectively). In children in the 1-4 years group the proportion of measles-mumps-rubella (MMR) vaccine related VAE was two times higher in PNI data when compared to local data $(27.4 \%$ and $13.5 \%$, respectively). In the 15-49 years group the proportion of VAE associated with YF vaccination was five times higher in local than in national data (50.8\% and $10.5 \%$, respectively). On the other hand, in this same age group the proportion of dTrelated VAE was three times higher at national than at local level.

Local adverse reactions comprised most adverse events related to BCG - lymphadenopathy, local abcess and ulcer -, DTP and dT vaccines - pain at injection site. The vaccines mainly related to systemic events were DTP - fever, hypotonic-hipporesponsive episode (HHE), convulsion and persistent crying - and YF vaccine (fever, arthralgia and hypersensitivity) (Table 4). A few rare events were reported with unusual frequency, such as aseptic meningitis associated to the YF vaccination campaign.

\section{Discussion}

The period analysed by this study were the first three years of implementation of the PNI's VAE Surveillance system and the results depicted a few problems of this process. Overall, data obtained in both local and national data are consistent regarding vaccines with high reactogenicity (DTP, BCG and MMR) and regarding the higher VAE frequency among
Table 4

Characteristics of VAE notified in Juiz de Fora, 1999-2001

\begin{tabular}{lrr}
\hline & $N$ & $\%$ \\
\hline Local events & 113 & \\
Abcess & 25 & 22.1 \\
Pain injection site & 67 & 59.3 \\
Limphadenopathy & 37 & 32.7 \\
Ulcer & 9 & 8.0 \\
Systemic events & 157 & \\
Arthralgia/arthritis & 11 & 7.0 \\
Persistent crying & 16 & 10.2 \\
Afebrile convulsion & 6 & 3.8 \\
Febrile convulsion & 13 & 8.3 \\
HHE & 30 & 19.1 \\
Fever & 110 & 70.1 \\
Hypersensitivity & 21 & 13.4 \\
Parestesias & 5 & 3.2 \\
Parotiditis & 7 & 4.5 \\
\hline
\end{tabular}

infants (younger than one year) and young adults for YF vaccine-related VAE.

Specific notification rates per vaccine based on local data were higher, possibly due to differences both in the numerator (number of VAE) and the denominator (number of applied doses). It is possible that there was an actual difference in VAE frequency or a higher sensitivity in the local system for detecting VAE, including differences in adopted criteria for diagnosing and recording cases. In fact, the proportional distribution of signs and symptoms suggested that less serious cases were notified in Juiz de Fora. Besides, the number of applied doses in the whole country is expected to be less accurate than in a middle-sized city.

Differences in patterns of detection/notification and in estimation of number of doses may not fully explain the huge discrepancy between the notification rates of BCG-related VAE obtained in nation-wide and Juiz de Fora data sets. In Juiz de Fora, VAE were concentrated in few vaccination units, which might indicate technical problems regarding BCG handling. In fact, the steady decrease of cases coincided with the training of new teams in BCG handling techniques.

Non-specific signs and symptoms such as fever and convulsion may have contributed to the higher frequency of DTPrelated VAE reported in Juiz de Fora. That is highlighted by the fact that HHE made up the highest proportion of VAE in children younger than 1 year in PNI data. The higher DPTrelated HHE frequency observed in Juiz de Fora - 26.9\% versus 3.3\% in VAERS (Vaccine Adverse Event Reporting System, CDC, USA) data [10] - can be partly explained by the previous occurrence of DPT-related VAE among HHE cases and the greater availability of DPaT vaccine (acellular pertussis) in the USA, where $84.4 \%$ of people who developed HHE had future doses suspended, compared to only $48.3 \%$ in Juiz de Fora. Besides, less specific events, such as fever, possibly related to other vaccines simultaneously taken (e.g. oral poliovirus and hepatitis B vaccines), could be attributed to DTP. 
MMR, measles-rubella, influenza, and YF vaccines had relatively higher VAE notification rates during vaccination campaign periods. Different from the other vaccines, mass vaccination campaign against yellow fever in 2001 was conducted only in State of Minas Gerais, probably explaining part of the higher apparent reactogenicity of that vaccine in Juiz de Fora, compared to the whole country. The relatively high frequency of MMR and measles-rubella vaccines related VAE was associated to a vaccination campaign in young women, but still the proportion of parotitis was higher than that observed in Rio de Janeiro campaign. The perception of VAE during mass vaccination campaigns can be altered by its concentration in a short period of time, by the heightened awareness by vaccinees and healthcare workers or by a temporal association with simultaneous outbreaks of other diseases.

The unusual record, not detected at the national level, of aseptic meningitis associated to YF vaccination was investigated by the Juiz de Fora's local Health Secretariat, using morbidity data to widen the ascertainment of cases. The investigation was limited by lack of laboratory resources, inaccuracy of data on doses applied by epidemiological week and of vaccine application date. Besides, the date of the beginning of VAE's symptoms, essential to accurately determine the time interval was not part of the notification form. The lack of such information limited rate estimates and hypothesis investigation in case-control or case series studies.

The investigation of possible VAE outbreaks, even if supported or conducted by the central level, depends mainly on the ability to detect and analyse data locally. Consolidated data for states and the whole country may reveal the occurrence of rare events or distribution patterns of events that point to differences between vaccine lots. The analysis of such data may not be of use to identify VAE related to faults in handling, maintaining and applying vaccines, since their effects are diluted in consolidated data. Besides, information generated by analysis at the central level may not arrive at the local level with the needed detail and swiftness to allow decision making. In a middle-sized city like Juiz de Fora, the epidemiological surveillance team is familiar with the vaccination units, their characteristics and professionals, which contributes to a faster understanding of events, as well as a more timely intervention.

Additional analysis comparing national data and analysis that are not part of routine surveillance allowed us to retrieve information relevant not only for the city but also to central state and national levels. Without local data analysis, the detection of and approach to possible VAE outbreaks may not be timely to prevent exposure of individuals to real risks and lead to misconceptions about the immunisation program. Moreover, local data analysis may provide more effective feedback to improve data quality. It seems that local and nation-wide data analysis are complementary.

Deficiencies in the formulation of notification forms as of 2001 were corrected by PNI. In spite of PNI guidelines as to how the forms should be filled and how data should be analysed, as well as global efforts to better define cases [9], inaccuracies are still found, reflecting difficulties in managing syndromes like HHE. For this and other cases, the recording of signs and symptoms rather than previously defined syndromes would allow healthcare workers who fill the forms, to deal with variations and incomplete or inconclusive cases without being forced to make a decision. Recording data by signs and symptoms might improve data analysis and comparability.

It may also be difficult to determine the level of seriousness of VAE, and that may affect the ability to compare data from different areas. Symptoms like fever, specially in infants, may be hard to evaluate as to their clinical meaning and to their relevance for VAE surveillance.

Aseptic meningitis cases linked to YF vaccine are an example of the potential and risks to data analysis at local level. On the one hand, the system was able to detect the concentration of cases in time and space, even using another database (morbidity database), but on the other it did not complete the investigation. As a result, the relation between that VAE and the YF vaccine is still unclear. In that sense, the revision of cases and data analysis stimulated the improvement of the local system, seeking to arrange data to perform case control or case series studies. The feedback to the system of information processed at higher levels, in spite of being recognised as part of surveillance activities, has not been fully implemented. Local initiatives such as the one described in this article may contribute to the improvement, by state and federal levels, of this element of the system.

\section{Acknowledgements}

Departamento de Epidemiologia, Secretaria Municipal de Saúde de Juiz de For a, Minas Gerais, Brazil.

\section{References}

[1] Singleton JA, Lloyd JC, Mootrey GT, et al. An overview of the vaccine adverse event reporting system (VAERS) as a surveillance system. Vaccine 1999;17:2908-17.

[2] Braun MM, Terracciano G, Salive ME, Blumberg DA, et al. Report of a US public health service workshop on hypotonichyporresponsive episode (HHE) after pertussis immunization. Pediatrics 1998;102(5).

[3] Rodrigues LC, Smith PG. Use of the case-control approach in vaccine evaluation: efficacy and adverse effects. Epidemiol Rev 1999;21(1):56-72.

[4] Chen RT, Orenstein WA. Epidemiologic methods in immunization programs. Epidemiol Rev 1996;18(2):99-117.

[5] Andrews NJ. Statistical assessment of the association between vaccination and rare adverse events post-licensure. Vaccine 2002;20:S49-53.

[6] Fine PEM, Chen RT. Confounding in studies of adverse reactions to vaccines. Am J Epidemiol 1992;136(2):121-35.

[7] Farrington CP, Nash J, Miller E. Case series analysis of adverse reactions to vaccines: a comparative evaluation. Am J Epidemiol 1996;143(11):1165-73. 
[8] MS (Ministério da Saúde). Manual de vigilância epidemiológica dos eventos adversos após vacinação, 1998.

[9] Bonhoeffer J, Kohl K, Chen R, Duclos P, Heijbel H, Heininger $\mathrm{U}$, et al. Standardized case definitions of adverse events following immunization (AEFI). Vaccine 2004;22(5-6):547-50.
[10] Duvernoy TS, Braun MM, The VAERS Working Group. Hypotonic-hyporresponsive episodes reported to the Vaccine Adverse Event Reporting System (VAERS), 1996-1998. Pediatrics 2000; 106(4). 